\title{
Editorial Metal Nanojoining
}

The objective of this Hot Topic edition of the Open Surface Science Journal is to group the pioneer works of the field of nanojoining into a single comprehensive volume.

Nanojoining is a new research area that is at the forefront of the nanotechnology craze, which is impacting all technical spheres of the society. Joining is a critical engineering discipline and is found on almost any components used in our everyday life. The miniaturisation of devices and the growing utilisation of advanced materials possessing non-equilibrium structures are the driving forces for the development of such technologies, as the conventional thermal load imposed would destroy the components to be joined or would simply cause the loss the desired microstructure.

Nanojoining takes its roots from the progress and innovations made through the development of the micro-joining field and is now incorporating significant phenomenon known to the nanoworld, such as increase surface energy, reduced activation energies. All these constraints are increasing the level of complexity for the development of joining procedures resulting in sounds interfaces. Parameters such as bonding temperature, time and pressureare becoming of paramount importance. It is however important to understand that this importance of this field will increase with the years, and thus overcoming the technological problem must be accomplish now to maintain the growth of nanotechnologies.

To demonstrate the worldwide importance of this field, the contributions presented in this collection were prepared by experts in the field coming from various countries, including Canada, China, India, Italy, Japan and the Unites States. This special scientific edition touches many aspects related to nanojoining. This works starts by an introduction to nanojoining (From Micro joining to Nanojoining), which present the evolution of the joining field to nanojoining and describes the principles challenges and applications. Secondly, the concept of extreme joining parameters, such as ultrafast processing time (Femtosecond laserinduced nanowelding: fundamentals and applications)or the utilisation of the increase surface energy to favor sintering at lower temperatures (Aggregation phenomena and electromagnetic amplification properties in silver nanoparticles joined through highly conjugated carbon chains, Evaluation of interfacial bonding utilizing $\mathrm{Ag}_{2} \mathrm{O}$-derived silver nanoparticles using TEM observation and molecular dynamics simulation, Effects of Joining Conditions on bondability of $\mathrm{Cu} / \mathrm{Cu}$ joint using $\mathrm{Cu}$ nanoparticle paste, Gold/Tin Oxide Nanocomposite by Nanojoining, and Low Temperature Bonding of $\mathrm{Cu}$ Metal through Sintering of Ag Nanoparticles for High Temperature Electronic Application). The special edition continues with the evaluation of new properties arising from nanojoing (Plasmonic properties of welded metal nanoparticles, Theoretical Investigations on Diffusion Induced Thermite Reactions of Core-Shell Aluminum/Palladium Nanoparticles) and finishes with concrete application of nanojoining (Nanowire Joining Methods, Development of Metastable Solidification Structures using the Electrospark, Adhesion Properties of Self-polymerized Dopamine Thin Film, Deposition Processing, and Evaluation of copper oxide-based interconnect materials).

This work is written to be a reference source to experts of the field, but done in a language and style ensuring accessibility to the general community interested in the field. This special issue was prepared to palliate to the spreading of emerging scientific information to form the first collection of work on nanojoining. It is hope that the breath and scope of this special issue will stimulate the development of new ideas that could contribute to the progression of the field.

Finally, the authors would like to acknowledge the many actors participating to the peer reviewing of the numerous manuscripts, the editing of the papers and the logistic around this special hot topic issue. Finally, we would like to thank the Open Surface Science journal for giving us the opportunity to produce this work.

\section{Anming Hu, Norman Zhou, Akio Hirose and Mathieu Brochu}

(Guest Editors)

Department of Mechanical and Mechatronics Engineering

University of Waterloo

200 University Avenue West

Waterloo

Ontario N2L 3G1

Canada

E-mail: a2hu@uwaterloo.ca

(C) Hu et al.; Licensee Bentham Open.

This is an open access article licensed under the terms of the Creative Commons Attribution Non-Commercial License (http://creativecommons.org/licenses/ by$\mathrm{nc} / 3.0 /$ ) which permits unrestricted, non-commercial use, distribution and reproduction in any medium, provided the work is properly cited. 